\title{
Human immunodeficiency virus infection and kidney disease
}

\author{
R Hilton \\ Consultant Nephrologist and Clinical Lead Nephrology, Abdominal Medicine and Surgery Directorate, Guy's Hospital, London, UK
}

\begin{abstract}
Since the introduction of highly active antiretroviral therapy (HAART) in 1996, mortality in patients with human immunodeficiency virus (HIV) infection has decreased markedly. As a result, morbidity from other chronic conditions such as kidney, liver and heart disease is increasing. This is in part as a natural consequence of ageing, and in part due to the higher risk of solid organ failure in these individuals. This higher risk is related to the co-morbidities associated with HIV infection and to the metabolic consequences of drug therapy. Kidney disease is an important cause of morbidity and mortality in patients with HIV infection and encompasses a range of clinical presentations including acute kidney injury, chronic kidney disease (CKD), and end-stage kidney disease. Black race is the most important predictor of kidney disease in HIV-infected persons due to both genetic and modifiable causes. HIVassociated nephropathy (HIVAN) is caused by viral infection of the renal epithelium and, although decreasing in incidence, remains the most common cause of CKD in HIV-infected persons. In addition to HIVAN, a wide spectrum of other histopathological presentations, many of which are immune complex-mediated, is increasingly recognised. Clinicians who treat these patients should be aware of the nephrotoxic potential of several commonly used medications, particularly antiviral agents, and also the need to dose-adjust medication in renal impairment. The increased life expectancy of patients with HIV and end-stage kidney disease has meant that kidney transplantation is increasingly an option. Results from carefully conducted prospective studies are promising, at least in the short term.
\end{abstract}

\author{
Correspondence to $\mathbf{R}$ Hilton \\ Guy's Hospital, \\ Great Maze Pond, \\ London SEI 9RT, UK
}

tel. $+44(0) 2071885691$

e-mail rachel.hilton@gstt.nhs.uk

KEYWORDS Antiretroviral therapy, HIV, HIVAN, kidney disease

DECLARATION OF INTERESTS No conflicts of interest declared.

\section{OVERVIEW}

The last decade has seen radical changes in the management of HIV. Whereas first generation antiretroviral drugs carried a high degree of toxicity, the introduction of newer agents with better efficacy and improved safety profiles has led to improved survival in $\mathrm{HIV}$-infected individuals, a marked decrease in AIDSrelated conditions, and a reduction in the incidence of HIV-associated nephropathy (HIVAN) as a cause of kidney dysfunction. However, there remain long-term adverse effects of medication, in some cases manifest as selective organ damage, but more commonly as a systemic accelerated ageing, so that cardiovascular disease, liver disease, chronic kidney disease (CKD), and non-AIDS-related cancers represent an increasing burden for HIV-infected individuals.

\section{HIV-ASSOCIATED KIDNEY DISEASE}

Kidney disease is now a leading cause of mortality in HIV. In 2009, data from a large prospectively studied cohort of over 16,000 HIV-infected patients across Europe, Israel and Argentina showed that kidney disease was the fourth leading cause of death among non-AIDS-related diseases, after malignancy, cardiovascular disease, and liver disease. Kidney disease was first reported in HIVinfected individuals in 1984, and initial reports identified both HIVAN and other kidney diseases. Highly active antiretroviral therapy (HAART) has altered the spectrum of kidney disease encountered in HIV-positive individuals so that the incidence of HIVAN has declined, whereas the burden of CKD due to diabetes, hypertension and drug toxicity has increased.

\section{HIV AND ACUTE KIDNEY INJURY}

HIV-infected persons have a higher risk for acute kidney injury (AKI) compared with their uninfected counterparts. Indeed, hospitalised HIV patients have a four- to five-fold increase in AKI in comparison with similarly matched non-HIV patients. In a prospective cohort study of over 50,000 HIV-infected persons in a US Registry, the incidence of dialysis-requiring AKI doubled between 2000 and 2006. Risk factors for AKI identified in this 
TABLE I Aetiology of acute kidney injury in HIV

\begin{tabular}{|c|}
\hline Pre-renal (actual or effective volume depletion) \\
\hline Renal \\
\hline $\begin{array}{l}\text { Acute tubular injury (nephrotoxic, ischaemic, pigment- } \\
\text { related) }\end{array}$ \\
\hline $\begin{array}{l}\text { Acute tubulo-interstitial nephritis (drugs - in over } 70 \% \text { of } \\
\text { cases, infection) }\end{array}$ \\
\hline $\begin{array}{l}\text { Glomerular disease (HIV-associated nephropathy } \\
\text { [HIVAN], HIV immune complex kidney disease } \\
\text { [HIVICK], immunoglobulin A [IgA] nephropathy, other } \\
\text { glomerulopathies) }\end{array}$ \\
\hline Vascular disease (thrombotic microangiopathy) \\
\hline Crystal nephropathy (uric acid, drugs) \\
\hline Post-renal (obstruction) \\
\hline
\end{tabular}

study included markers of kidney health such as preexisting CKD and proteinuria, and HIV-related factors such as low serum albumin, low body mass index (BMI), low CD4 count and high viral load (Table I).

Although HIV-associated glomerulopathies are widely recognised renal syndromes, AKI in HIV-infected patients may result from a diverse number of causes, many of which are also seen in non-HIV patients, including pre-renal AKI, nephrotoxic and ischaemic acute tubular injury, and obstructive uropathy. However, some syndromes are more common in patients with HIV and some are characteristic. Paradoxically, although HAART is used to treat HIVAN, the drugs themselves can cause kidney damage, including proximal tubular dysfunction, acute tubular injury, and nephrolithiasis. Causes of drug-induced kidney disease include several well-known tubular toxins such as aminoglycosides, amphotericin, cidofovir, and foscarnet, crystal-induced

\section{TABLE 2 Drug toxicity in HIV}

\begin{tabular}{|l|l|l|}
\hline $\begin{array}{l}\text { Acute tubular } \\
\text { injury }\end{array}$ & $\begin{array}{l}\text { Acute tubulo- } \\
\text { interstitial } \\
\text { nephritis }\end{array}$ & $\begin{array}{l}\text { Crystal } \\
\text { nephropathy }\end{array}$ \\
\hline Adefovir & $\begin{array}{l}\text { Non-steroidal } \\
\text { anti-inflammatory } \\
\text { drugs (NSAIDs) }\end{array}$ & Aciclovir \\
\hline Aminoglycosides & Co-trimoxazole & Atazanavir \\
\hline Amphotericin B & Dapsone & Foscarnet \\
\hline Cidofovir & Quinolones & Indinavir \\
\hline Co-trimoxazole & Atazanavir & Sulfadiazine \\
\hline Foscarnet & Abacavir & Uric acid \\
\hline NSAIDs & Indinavir & \\
\hline Pentamidine & Omeprazole & \\
\hline Radiocontrast & Beta-lactams & \\
\hline Tenofovir & Cocaine & \\
\hline
\end{tabular}

renal injury due to indinavir and sulfadiazine and other conventional nephrotoxins such as radioiodine. Acute tubulo-interstitial nephritis is also relatively common and may relate to infection but is most often druginduced (Table 2).

Three drugs feature prominently among antiretroviralrelated kidney disease: indinavir, atazanavir, and tenofovir. Indinavir and atazanavir are potent protease inhibitors (PI) and are the PI most commonly associated with nephrotoxicity. The main feature is crystalluria, which may be asymptomatic or manifest as flank pain and dysuria. The stones are radiolucent, so imaging studies may show obstruction without calculi. Insidious renal impairment may develop due to tubular injury, interstitial inflammation and scarring. Risk factors include dehydration, low BMI, co-infection with hepatitis viruses, and co-administration of other tubular toxins. Indinavir is now rarely prescribed because of its stone-forming tendency, whereas atazanavir is the first choice PI in patients who need to take this class of drugs, and some patients are encouraged to switch to it. During treatment with indinavir or atazanavir, high fluid intake and close monitoring for pyuria are recommended.

Tenofovir was the first nucleotide reverse transcriptase inhibitor approved for treatment of HIV infection. Concern over antiretroviral drug-induced toxicity has largely focused on this drug, although the overall risk of nephrotoxicity is low and most often seen in patients with predisposing kidney disease or co-morbidities such as diabetes or hypertension. Tenofovir is excreted by the kidney via a combination of glomerular filtration and active tubular secretion. Several case reports and observational studies have found evidence of potential kidney toxicity with tenofovir, mostly a proximal tubulopathy with Fanconi syndrome and nephrogenic diabetes insipidus. Long-term monitoring of kidney function and markers of tubular dysfunction should be undertaken in patients taking tenofovir, particularly in those with co-morbidities such as pre-existing CKD, hypertension or diabetes, or predisposing factors such as old age or co-administration of other nephrotoxic drugs.

\section{HIV AND CHRONIC KIDNEY DISEASE}

Chronic kidney disease is relatively common in HIVinfected persons. A study conducted in an urban US HIV population found evidence of CKD in a quarter of patients, mostly mild to moderate. Patients with CKD were more likely to be black, to be older, and to have AIDS, low CD4 counts, and higher HIV viral loads. There was also an association of CKD with hypertension, diabetes, or both. The prevalence of CKD and the likelihood of progression are both significantly higher in HIV-infected subjects compared with HIV-negative subjects. In a Ugandan community-based cohort study of 
over I,800 HIV-infected and age- and sex-matched HIVnegative controls, HIV-infected patients were twice as likely to have evidence of mild or moderate reduction in glomerular filtration rate (GFR). During follow-up there was a $50 \%$ higher risk of decline in GFR in HIV-infected versus non-infected subjects. Similar observations have been made in industrialised countries.

Black race is the most important determinant of kidney disease prevalence in HIV-infected persons due to both genetic and modifiable causes. In a US study looking at CKD incidence and progression to end-stage renal disease (ESRD) in almost 4,000 HIV-infected subjects, black subjects were at twice as high a risk for incident CKD than white subjects and had a six-fold more rapid decline in GFR than the white subjects with a markedly faster rate of developing ESRD. This was irrespective of the presence of HIVAN. These data suggest that racial disparities in HIV-related ESRD can be explained at least in part by a more aggressive natural disease history in black subjects. Recent data suggest that this may in part be due to polymorphisms in the apolipoprotein L-I gene on chromosome 22, which confer susceptibility to kidney disease in people of African ancestry but are also more effective in lysing certain subspecies of Trypanosoma brucei, the causative agent of sleeping sickness. This gives a plausible explanation for why this genotype has been selected in persons of African descent.

Expert guidelines recommend screening and early identification of CKD in patients with HIV, and this should prompt nephrology referral, initiation of HAART, and tight control of co-morbid diabetes and hypertension (if present). Medication doses should be adjusted for the level of kidney function, with particular attention to nucleoside reverse transcriptase inhibitors (see below). HIV-associated nephropathy is probably the best known example of the specific manifestations of CKD in HIV, but a wide spectrum of pathology is possible and indeed frequently seen. In addition, HIV is linked to disorders such as thrombotic microangiopathy and glomerulonephritis associated with immune complex deposition and hepatitis B or C co-infection. Highly active antiretroviral therapy also contributes indirectly to CKD by increasing the risk of diabetes and hypertension.

\section{HIV-ASSOCIATED NEPHROPATHY}

This is the most common cause of CKD, occurring in 12 to $15 \%$ of HIV-infected black patients. Classically this is a late manifestation of HIV infection associated with low CD4 counts and high viral loads and characterised by nephrotic-range proteinuria, increased kidney echogenicity on ultrasound, lack of hypertension, or oedema and progressive kidney failure. Histological examination of the kidneys reveals collapsing focal and segmental glomerulosclerosis, tubular epithelial atrophy with microcystic dilatation of the tubules and lymphocytic interstitial infiltration. HIV viral replication in kidney cells is a prerequisite for disease development, circulating viral RNA is associated with kidney disease progression, and effective inhibition of viral replication improves kidney function and prolongs kidney survival.

\section{HIV IMMUNE COMPLEX-MEDIATED KIDNEY DISEASES}

The term HIVICK represents a variety of histological entities with common aetiological features including deposited immune complexes containing HIV antigens, characteristic cytokine expression profiles, genetic factors, inflammatory infiltrates, and the development of kidney scarring. Among the many reported pathologies immunoglobulin A $(\lg A)$ nephropathy is relatively common and is believed to result from immune complexes that contain HIV antigens. Lupus-like nephropathy typically presents with microscopic haematuria, proteinuria and renal impairment with relatively rapid progression to kidney failure. Histologically there may be diffuse or focal proliferative changes, membranous nephropathy, crescent formation, and tubule-interstitial scarring. Optimal management is unclear and some series have failed to show that HAART influences progression to ESRD.

\section{HIV AND END-STAGE RENAL DISEASE}

Despite the decreasing incidence of HIVAN due to early initiation of HAART, kidney disease remains highly prevalent in HIV-infected individuals, many of whom will progress to ESRD. Indeed, the number of HIV-infected patients in the US dialysis programme tripled between 1985 and 1988, although it is now stable or decreasing. The highest risk subset is young black males; HIVAN remains the third leading cause of ESRD among African Americans aged 25-64 years, after diabetes and hypertension. The prevalence of HIV is between I and $2 \%$ of the US ESRD population, the prevalence geographically reflecting the national distribution of AIDS and HIV infection. With effective therapy, survival in dialysis-dependent HIV-infected individuals is now similar to that of HIV-uninfected persons. The increased life expectancy of patients with HIV and ESRD has meant that kidney transplantation is increasingly an option, and results from carefully conducted prospective studies are promising, at least in the short term. However, rates of acute allograft rejection are increased in these patients, co-infection with HIV and hepatitis $C$ virus (HCV) is a major concern in terms of treatment options and long-term effects on progression of $\mathrm{HCV}$, and there are multiple drug interactions between HAART and immunosuppressants which mandate a careful multi-disciplinary approach to management. 
Effective dosing of HAART in patients with impaired kidney function and ESRD is challenging. In general protease inhibitors and non-nucleoside reverse transcriptase inhibitors are metabolised in the liver and do not require dose adjustment in renal impairment. However, most nucleoside/tide reverse transcriptase inhibitors are excreted unchanged in the urine and require careful dose adjustment and/or extension of the dosing interval in renal impairment. This can be problematic for fixed dose drug combinations. Specialist advice is recommended.

\section{FURTHER READING}

I Campbell LJ, Ibrahim F, Fisher $M$ et al. Spectrum of chronic kidney disease in HIV-infected patients. HIV Med 2009; 10:329-36. http:// dx.doi.org/I0.IIII/j.|1468-I293.2008.0069I.x

2 Frassetto LA, Tan-Tam C, Stock PG. Renal transplantation in patients with HIV. Nat Rev Nephrol 2009; 5:582-9. http://dx.doi. org/10.1038/nrneph.2009.140

3 Li Y, Shlipak MG, Grunfeld $C$ et al. Incidence and risk factors for acute kidney injury in HIV infection. Am J Nephrol 20I 2; 35:327-34.

4 Maggi P, Bartolozzi D, Bonfanti $P$ et al. Renal complications in HIV disease: between present and future. AIDS Rev 2012; 14:37-53.

\section{Highlights}

- Kidney disease is increasingly common in patients with human immunodeficiency virus (HIV).

- African ancestry defines a high-risk subgroup of patients.

- $\quad$ Highly active antiretroviral therapy (HAART) has impacted favourably on the incidence and prognosis of HIV-associated nephropathy (HIVAN), but drug toxicity remains a key concern.

- Patient survival on dialysis is now similar in HIVand non-HIV-infected patients.

- Kidney transplantation offers effective therapy for patients with HIV infection and end-stage kidney disease but requires a careful multi-disciplinary approach.

5 Panel on Antiretroviral Guidelines for Adults and Adolescents. Guidelines for the use of antiretroviral agents in HIV-I-infected adults and adolescents [Internet]. Department of Health and Human Services: 2012 [cited 2013 March 3]. Available at: http://aidsinfo. nih.gov/guidelines

6 Phair J, Palella F. Renal disease in HIV-infected individuals. Curr Opin HIV AIDS 20II; 6:285-9. http://dx.doi.org/I0.1097/COH.0b0I3e $3283476 \mathrm{bc} 3$

7 Roe J, Campbell LJ, Ibrahim F et al. HIV care and the incidence of acute renal failure. Clin Infect Dis 2008; 47:242-9. http://dx.doi. org/10.1086/589296 


\section{SELF-ASSESSMENT QUESTIONS}

I. Which ONE of the following DOES NOT cause acute kidney injury in patients with HIV?

A. Salmonella gastroenteritis.

B. Nitrofurantoin.

C. Haemolytic uraemic syndrome.

D. Indomethacin.

E. Staphylococcal endocarditis.

2. Which ONE of the following medications DOES NOT cause acute tubulo-interstitial nephritis in HIV?
A. Omeprazole.
B. Dapsone.
C. Maraviroc.
D. Co-trimoxazole.
E. Atazanavir.

3. A 26-year-old Black-African man is found to be HIV positive and to have marked proteinuria (I I g/day), renal impairment (eGFR $26 \mathrm{ml} / \mathrm{min}$ per $1.73 \mathrm{~m}^{2}$ ) and protein (3+) on urinalysis. The kidneys are of normal size and are hyperechoic on ultrasonography. Which ONE of the following statements is most correct?

A. A lesion other than HIV-associated nephropathy will likely be found on kidney biopsy.

B. He is unlikely have a mutation at the apoprotein L-I gene on chromosome 22.

C. He is likely to have severe peripheral oedema.

D. Treatment with highly active retroviral agents will not likely influence the course of his kidney disease.

E. He is likely to be normotensive.
4. Kidney transplantation in patients with HIV infection is associated with which ONE of the following

A. A low rate of T-cell-mediated allograft rejection in association with low CD4+ $T$ cell counts.

B. Better graft and patient survival than in non-HIV-infected controls.

C. Evidence of accelerated HIV disease progression.

D. A $15 \%$ risk of recurrence of HIV-associated nephropathy.

E. Higher than expected graft rejection rates.

5. Regarding mortality in HIV, which ONE of the following statements is MOST correct?

A. Kidney disease is the leading cause of death among nonAIDS-related diseases in HIV-infected patients in Europe.

B. Antiretroviral therapy has dramatically reduced HIVassociated opportunistic infections but has not impacted upon overall mortality.

C. Coronary artery disease is the leading cause of death or hospitalisation in HIV-infected patients in the US.

D. US Centers for Disease Control and Prevention reported a doubling of the incidence of opportunistic infection in HIV-infected patients in the US between 2000 and 2006.

E. Patients with HIV are more likely to die of non-AIDSrelated malignancy than of kidney failure.

This paper was originally published as part of the Renal module in the RCPE Online Continuing Medical Education Programme. Online CME, including the anwers to these questions, is available to Fellows and Members at: http://www.rcpe.ac.uk 